\title{
Post exposure prophylaxis of HIV transmission after occupational injuries in Queen Elizabeth Central Hospital, Blantyre, Malawi, 2003 - 2008
}

\author{
Gerrit C van der Maaten ${ }^{1,2}$, Mulinda Nyirenda ${ }^{1}$, Micheal J Beadsworth ${ }^{3}$, Alex Chitani' \\ Theresa Allain ${ }^{1}$, Joep J van Oosterhout ${ }^{1}$ \\ 1. Department of Medicine, University of Malawi College of Medicine, Blantyre, Malawi \\ 2. Erasmus University, Rotterdam, Netherlands \\ 3. Tropical and Infectious Disease Unit, Royal Liverpool University Hospital, Liverpool, UK
}

\begin{abstract}
Health care worker (HCW) in Malawi may acquire HIV infection through occupational injuries, in particular since HIV prevalence among inpatients and incidence of occupational injuries are high. A post exposure prophylaxis (PEP) programme for occupational injuries at Queen Elizabeth Central Hospital (QECH) commenced in 2003. We performed an audit of this programme from 2003 through 2008. 203 Occupational injuries were reported. The majority were needle stick injuries $(76.3 \%)$. Half of the clients were in a training position. A dual ART regimen was most frequently prescribed. Triple therapy use increased over time and was more frequent in expatriate students. Many nurses and clinical officers were not fully vaccinated for HBV. Based on previous incidence data, occupational injuries were likely to be underreported. Data on side effects were incomplete, however PEP discontinuation due to side effects occurred only twice. Follow up visits were poorly attended, therefore the efficacy of PEP could not be evaluated.

Prevention efforts for occupational injuries should be increased and specifically target HCWs in training positions. Measures to improve quality of the PEP programme include effective publicity campaigns, compulsory Hepatitis B vaccination and active tracing of HCWs who default follow up after PEP.
\end{abstract}

\section{Introduction}

After an occupational injury, healthcare workers (HCW) run a risk of infection with HIV. The WHO estimates that approximately 3 million percutaneous exposures occur worldwide each year among HCWs ${ }^{1}$. The chance that HCWs acquire HIV through occupational injuries may be greater in Malawi and other countries in sub-Saharan Africa than in other parts of the world. Firstly, the HIV prevalence in the community is high and is considerably higher still among hospital inpatients, as many are admitted due to HIV related diseases. Secondly, available data from sub-Saharan African countries show that standard precautions to prevent occupational injuries are often poorly implemented ${ }^{3}$, resulting in a high incidence of occupational injuries ${ }^{1}$.

The estimated risk of HIV transmission is $0.3 \%$ after a needle stick injury and $0.09 \%$ after a mucous-membrane exposure ${ }^{4}$. Risks of other types of exposures are unknown. Several factors are associated with higher HIV transmission risk $^{5}$ : deeper injuries, visibility of blood on the sharp device, a procedure involving a needle that was in the source patient's artery or vein, and a source patient who died of AIDS within two months after the accident. These risk factors reflect the volume and HIV concentration of the transmitted blood. Although the absolute risk is small, the occupational injury often causes stress and anxiety ${ }^{6}$.

Few data exist about the efficacy of PEP after occupational injuries. In a retrospective study from France, Italy, the United Kingdom and the United States prophylactic treatment with zidovudine resulted in a risk reduction of HIV transmission of $81 \%{ }^{5}$. Evidence of benefit of PEP can also be assumed by extrapolation from animal experiments ${ }^{7}$ and prevention of mother to child transmission studies 8 .

Free of charge anti-retroviral therapy (ART) has become widely available in Malawi since 2004, in the context of a successful national ART scale-up programme that includes antiretroviral drug provision for PEP after occupational injuries. A PEP programme in the Queens Elizabeth Central Hospital (QECH) in Blantyre was introduced in 2003. Very few studies are available concerning PEP programmes in sub-Saharan Africa. We evaluated the PEP programme of QECH in 2004 and showed that despite an adequate logistical organization, major shortcomings were present: insufficient awareness of the programme among HCWs, and poor follow up after the first consultation for $\mathrm{PEP}^{10}$.

We performed an audit to describe features of the PEP programme in QECH from 2003 through 2008 and determine whether initial deficiencies had been overcome.

Figure 1. Number of reported occupational injuries in QECH per year ${ }^{8}$

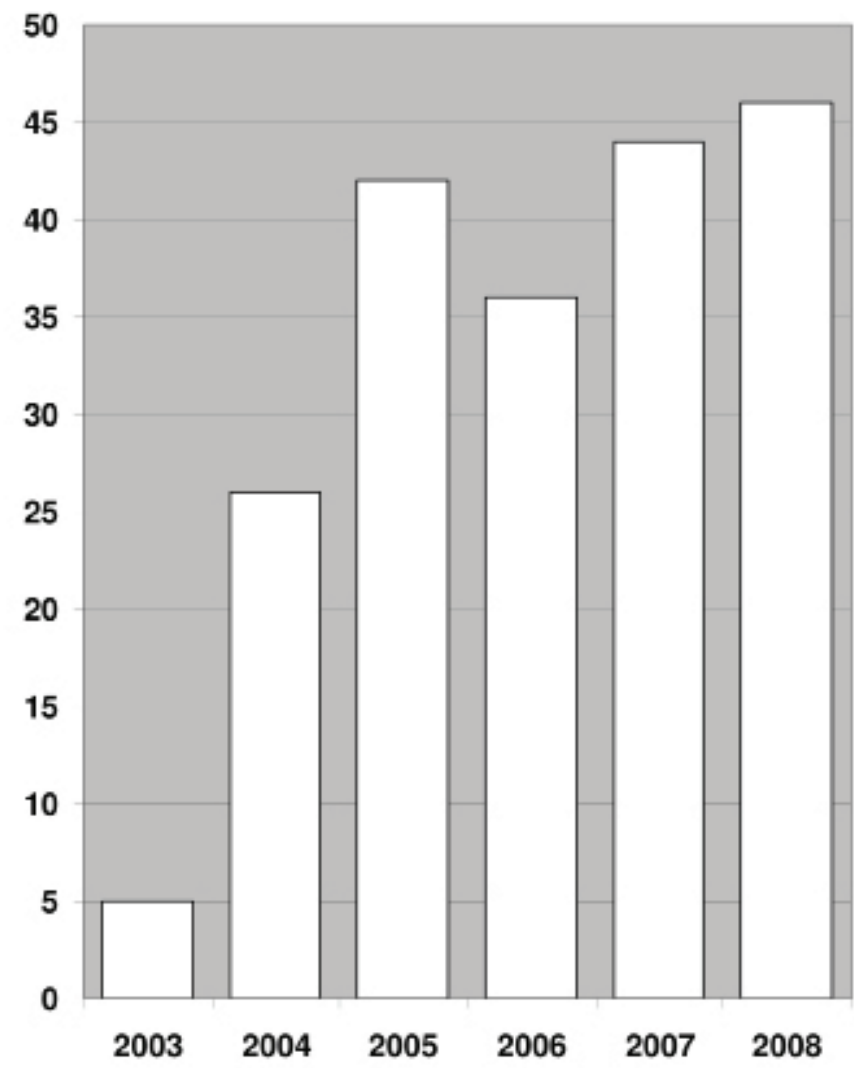




\section{Methods}

Study site

The QECH is a primary, secondary and tertiary care hospital in Blantyre, the largest city in the Southern Region of Malawi. It is the teaching hospital of the College of Medicine (the only medical school in Malawi) and of colleges that train nurses and clinical officers. The hospital admits 70,000 patients per year ${ }^{11}$. HIV prevalence among inpatients in $\mathrm{QECH}$ ranges from $36.0 \%$ in the surgical wards to $70.0 \%$ in the medical wards ${ }^{12}$.

\section{$P E P$ services offered at QECH}

The Department of Medicine of the College of Medicine initiated the PEP programme to prevent HIV infection after occupational injuries in QECH in 2003. There are also PEP services for women and children who have been raped, but these were not included in this audit. PEP service is available 24 hours per day, 7 days per week. If the occupational injury occurs outside office hours, initial management and "starter pack" supply takes place at the intensive care unit, with subsequent follow up at the Department of Medicine. For each client seeking advice for PEP, a standard proforma is filled out. These PEP files are stored in a lockable metal filing cabinet in the Department of Medicine.

National ART Guidelines ${ }^{9}$ for the use of PEP have been implemented with adaptations to the local situation at QECH. Clinicians assess the occupational injury to classify the risk of HIV transmission either as high or low. Percutaneous injuries with hollow needles and splash accidents onto mucous membranes with larger volumes of blood are classified as high-risk accidents.

Percutaneous injuries with solid needles, exposures to other body fluids than blood, and exposures to the non-intact skin are classified as low risk. Clients with a high-risk classification and those with an exposition to body fluids from a source person on a standard fist-line ART regimen receive triple therapy (zidovudine and lamivudine plus a protease inhibitor such as ritonavir boosted lopinavir, if available). Otherwise dual therapy with zidovudine and lamivudine is indicated. The antiretroviral drugs are provided free of charge. Appointments for 4 follow up visits are scheduled (after two weeks, and one, three and six months). Full blood counts are checked at baseline, and the first two follow up visits. Side effects are documented and HIV tests are scheduled after 3 and 6 months.

\section{Data collection and analysis}

A retrospective audit was performed of all the files that were available from May 2003 through December 2008. The data extracted were occupation, department, HIV status and HBV vaccination status of the HCW, as well as the time, date and detailed description of the exposure, the HIV status of the source, the time between reporting and starting PEP, the prescribed regimen, the reported side effects and results of HIV tests. To obtain data on the number of nurses, student nurses and medical students working in the hospital, we interviewed nurses in charge of each inpatient ward. Data were entered in a database in SPSS 12.0 (SPSS Inc., Chicago, IL, USA). Categorical variables were compared using Chisquare or Fisher's exact tests, continuous data using KruskalWallis tests and Student's t-tests.

\section{Results}

From the start of the PEP program in QECH in May 2003, through 2008, 203 occupational injuries were reported.
Figure 2. Type of injury

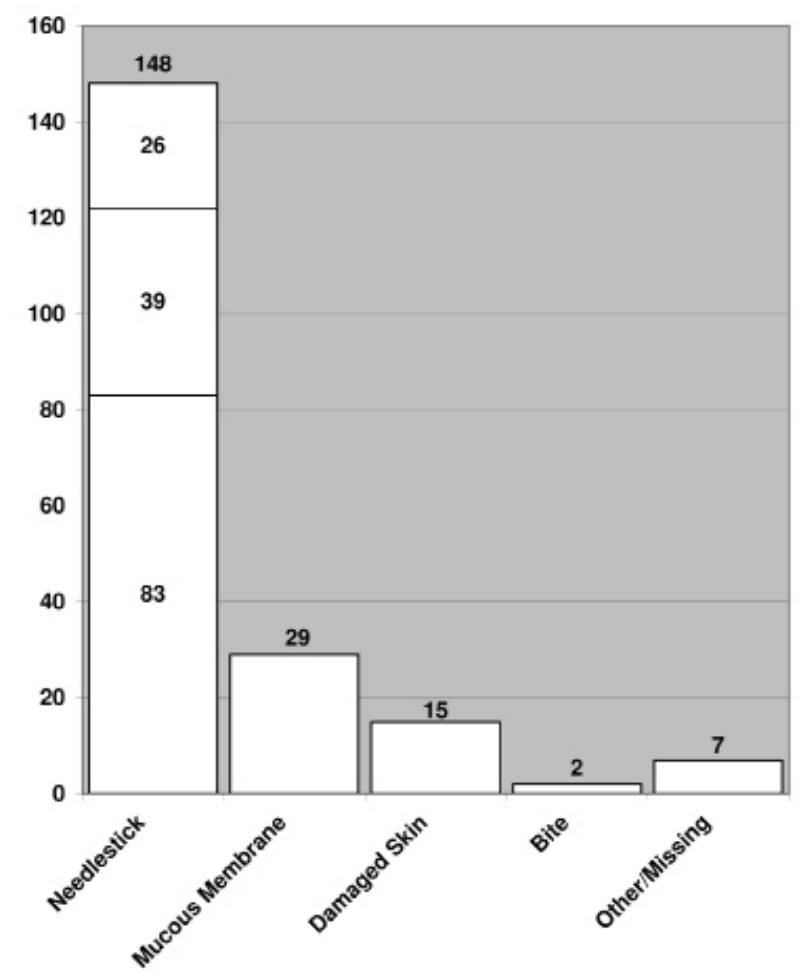

83 of the needle stick injuries were with a hollow needle, 39 with a solid and 26 with an unknown type of necdle.

Two files were excluded from analysis due to insufficient documentation. In 2003 there were only 5 reported occupational injuries, and since 2005 the number has remained stable around 40 per year (figure 1 ). The majority $(76.3 \%)$ were needle stick injuries (figure 2). Most occurred in the obstetrics and gynaecology department, and very few were reported outside the clinical departments (table 1). The mean age (standard deviation) of the clients was 27 years (11.1). The most recorded occupation was medical student and of whom more than $40 \%$ were expatriate elective students.

Overall, $50.7 \%$ of the HCWs were in a training position (table 2). Nearly three-quarters of the occupational injuries happened within office hours (8.00h-17.00h), the highest number occurred between $16.00 \mathrm{~h}-17.00 \mathrm{~h}$ (figure 3).

PEP was initiated in $168(83.6 \%)$ cases. In 33 cases PEP was not indicated: the source was tested HIV-negative in 16, the accident had happened too long ago in two, one client was tested HIV positive, and another was lost to follow up before risk assessment. In 11 cases it was decided that the nature of the injury made the risk of HIV transmission insignificant. In the remaining four cases the reason why PEP was not started could not be determined. Of the 168 clients who initiated PEP, five stopped within 10 days because the source was tested HIV negative, two failed to attend for additional medication after a starter-pack supply and one was tested HIV positive. Two further clients stopped PEP because of sideeffects, and one because he decided that no real occupational injury had happened. From 25 clients who started PEP, the risk classification of the accident could not be established from the case notes. Of the remaining 143 cases, 42.6\% were determined as low risk, and $57.4 \%$ as high risk. A dual ART regimen was prescribed in $85.2 \%$, triple therapy in $14.8 \%$ of the cases. There was a significant increase in triple therapy over time, from $9.5 \%$ in 2005 to $21.7 \%$ in 2008 ( $\mathrm{p}<0.01)$. 
The percentage of expatriate elective students that received triple therapy was higher compared to other groups $(70.0 \%$ vs. $12.5 \% ; \mathrm{p}<0.001)$, differences between other groups were not significant (table 3 ).

The mean duration between the occupational injury and PEP initiation was 7.66 hours (range: $0.3-72$ hours, SD 13.74), in 86 cases the duration was not reported. PEP was started within two hours by the majority $(55.7 \%)$ of clients, and $96.5 \%$ started within 48 hours. There were no significant differences in time between occupational injury and PEP start between the occupations of the clients (data not shown).

Very few clients attended follow up visits. Only $25.2 \%$ of the clients who started PEP attended the first follow up visit, $12.6 \%$ the second, $6.3 \%$ the third, and $1.9 \%$ the fourth. The most common side effects reported at the first follow up visit $(\mathrm{n}=41)$ were nausea $(36.6 \%)$, headache $(14.6 \%)$, fatigue $(12.2 \%)$, vomiting $(7.3 \%)$, and abdominal pains $(7.3 \%)$. Side effects led to discontinuation of PEP in only two cases. Only 30 full blood counts were reported at the first visit and 13 at the second. Anemia and neutropenia were rare and never led to PEP interruption. Adherence to the PEP regimen was recorded during the second follow up visit from 19 clients: 13 had completed the full course, 6 had missed at least one dose. A total of $12 \mathrm{HIV}$ tests results were recorded, all were negative.

The Hepatitis-B virus (HBV) vaccination status of $55 \mathrm{HCWs}$ was not documented, $77(38.3 \%)$ were fully vaccinated, 18 (9.0\%) partly HBV vaccinated, and $51(25.4 \%)$ not vaccinated. Among doctors plus medical students the proportion being fully vaccinated was highest $(87.9 \%)$, followed by nurses plus nurses in training $(31.6 \%)$, and clinical officers plus clinical officers in training $(18.8 \%)(\mathrm{p}<0.001)$. There was not a clear trend in time of the proportion of HCWs being nonvaccinated, and it remained high in 2008 (one-third).

Interviews with nurses in charge of the inpatient wards in December 2008 indicated that 2 to 6 nurses per ward were present during day shifts and 2 to 3 during night shifts. In addition there were 2 to 8 student nurses during day shifts, but only for part of the year. Medical students had clinical rotations on the wards throughout the year, and did regular evening and night on calls. On average 6 students were attached to one ward.

Figure 3. Number of occupational injuries per hour of the day

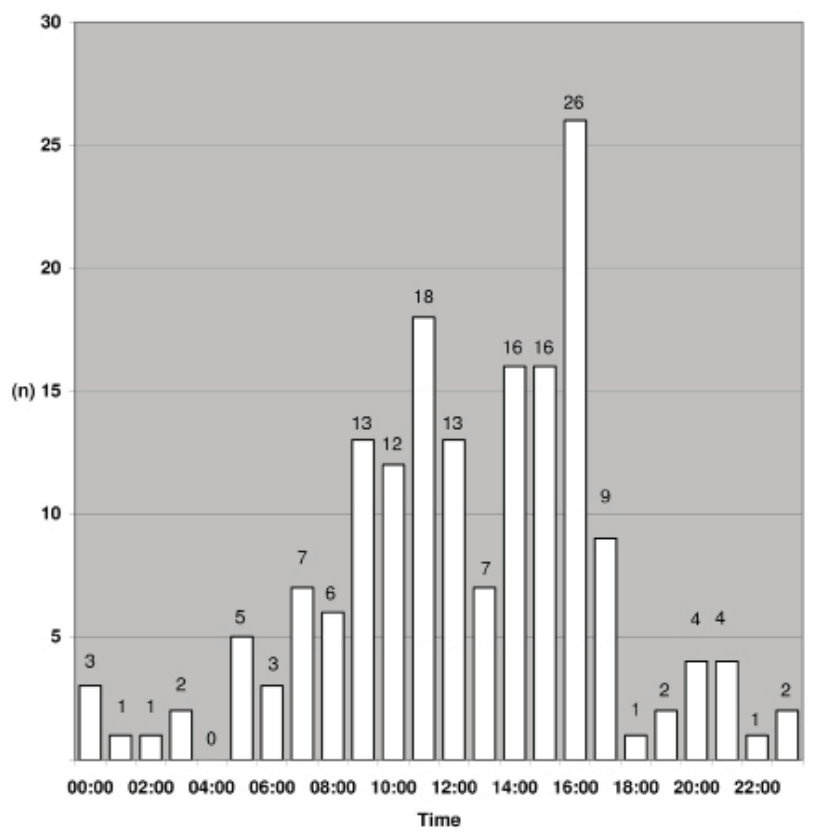

Table 1. Department of the HCW who sustained the occupational

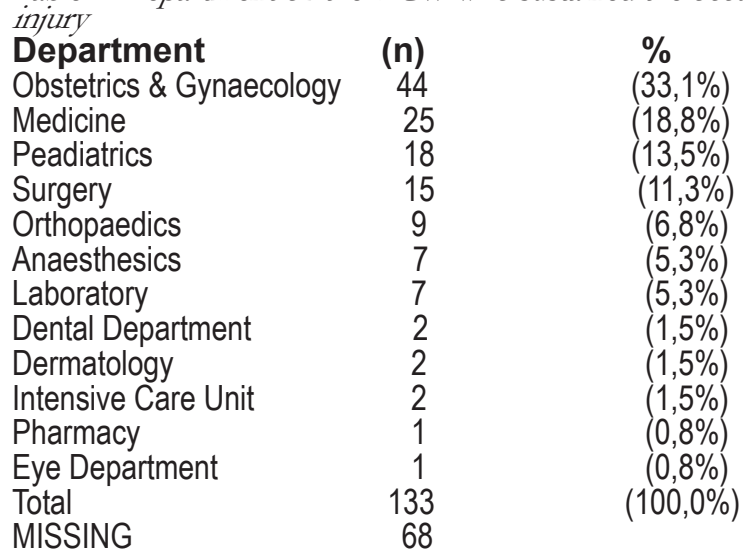

Table 2. Occupation of the clients versus type of PEP regimen

$\begin{array}{lcccc}\text { Occupation } & \text { Dual Therapy } & \text { Triple Therapy } & \text { MISSING } & \text { Total } \\ \text { Nurse } & 19 & 2 & 6 & 27 \\ \text { Doctor } & 17 & 6 & 8 & 31 \\ \text { Student Nurse }^{*} & 17 & 1 & 1 & 19 \\ \text { Medical Student }^{*} & 15 & 2 & 3 & 20 \\ \text { Medical Intern }^{*} & 11 & 2 & 1 & 14 \\ \text { Clinical Officer } & 9 & 0 & 1 & 10 \\ \text { Clinical Officelntern* } & 6 & 1 & 2 & 9 \\ \text { Exp. ElectiveStudent } & 3 & 7 & 4 & 14 \\ \text { No HCW } & 4 & 0 & 1 & 5 \\ \text { Cleaner } & 0 & 0 & 1 & 1 \\ \text { Missing } & 31 & 2 & 18 & 51\end{array}$

31

51

\section{Discussion}

After an initial rise in the number of reported occupational injuries, it stabilized to around 40 cases per year. It is likely that occupational injuries were underreported, since the incidence of occupational injuries in QECH among nurses was estimated at 1.5 per nurse per year10 and around 250 nurses plus a similar number of clinical officers, doctors, medical students and interns were working in QECH during the observation period ${ }^{11}$. A study from Tanzania ${ }^{15}$ also described underreporting of occupational injuries. A qualitative study from Kenya ${ }^{16}$ demonstrated that the main reason for poor uptake of PEP was fear of getting tested for HIV. Other reasons why HCWs may not report occupational injuries are unawareness of the existence of a PEP service, underestimation of the risk of HIV transmission, and unwillingness to take antiretroviral drugs. Needle sticks injuries were by far the most commonly reported occupational injuries in QECH, which is similar in western countries ${ }^{5}$, as well as in South Africa ${ }^{17}$, India ${ }^{18}$, and Thailand ${ }^{19}$.

Notable is that half of the occupational injuries in QECH happened in persons in training positions. In other studies, most cases were among nurses and medical interns 18-20, however this was highly dependent on the type of health care facility. QECH is a teaching hospital, and the number of students has increased steadily over time. Occupational injuries may also be common among students due to the fact that they perform invasive medical procedures, such as venous, ascites and lumbar punctures that are often done at later stages of the medical training in other parts of the world.

Most occupational injuries happened at the end of the working day, probably because of a combination of high work pressure and fatigue. Awareness of the increased risks 
at specific times of the day may help to prevent occupational injuries.

Only 16 source patients were found to be HIV negative. This number is lower than in other studies, ${ }^{18,19,21}$ which is not surprising given the high HIV prevalence among inpatients in QECH.

Another explanation may be that an increasing percentage of inpatients has a known HIV status, and HCWs may decide not to report occupational injuries sustained from known HIV negative source patients.

PEP was withheld on one occasion because the HCW was HIV positive. This number was expected to be higher, because the HIV-prevalence among HCWs in Malawi is high $^{2}$. It is possible that HCWs who know their positive HIV status choose not to report occupational injuries.

The majority of HCWs started PEP within 2 hours and nearly all within 48 hours after the occupational injury. These results are slightly lower than in a study from Thailand where $77 \%$ started PEP within 2 hours. We found no data on the causes of delay in starting PEP neither in this audit nor in literature.

Full Hepatitis B vaccination prevalence among HCWs was low compared to western countries, however studies from Kenya $^{16}$ and Egypt ${ }^{22}$ showed even lower rates $(15.0 \%$ and $15.8 \%$, while in a report from West Africa ${ }^{20}$ coverage was similar to QECH. In October 2006 a HBV vaccination campaign for nurses and clinical officers took place, however this did not appear to have had a major impact on the vaccination coverage among clients of the PEP programme. Complete vaccination of all HCWs and students needs to remain a priority in the light of the high $\mathrm{HBV}$ prevalence among inpatients, ${ }^{23}$ the high chance of $\mathrm{HBV}$ transmission during an occupational injury, and the safety and efficacy of the $H B V$ vaccine ${ }^{24}$.

Most clients in QECH received dual therapy PEP, independent of the HIV transmission risk classification. Protease inhibitors were not available until 2006 and the overall percentage of clients who received triple therapy was low. To comply with guidelines and cater for increasing number of exposures to source patients who are on a first line ART regimen (in which case triple therapy PEP including a protease inhibitor is required because of the potential presence of resistance to zidovudine and lamivudine), protease inhibitors should become more readily available for PEP. Most expatriate elective students received triple therapy, even if the risk assessment was low, because they were provided with PEP medication kits from their countries of residence.

Follow up attendance after initial assessment and provision of PEP medication was very low, and therefore we could not determine the efficacy of the PEP programme to prevent HIV transmission.

Side effects were common but rarely severe enough to cause discontinuation of PEP. In the only other study of PEP from sub-Saharan Africa that reported on a smaller number of occupational injuries, HCWs in Western Kenya also declined scheduled follow up visits frequently ${ }^{25}$. Data on side effects in our audit were too incomplete to justify recommendations for pre-emptive symptomatic therapy.

Our major findings, namely probable underreporting of occupational injuries, poor attendance of follow up visits and high percentage of $\mathrm{HCW}$ s without $\mathrm{HBV}$ vaccination, were also reported in our earlier audit in 2004. More effective publicity and awareness campaigns (fig 4), active tracing of HCWs who default follow up, and compulsory HBV vaccination at the start of the training or employment of all HCWs may improve the quality of the current PEP programme. Efforts to prevent occupational injuries should be increased and specifically target HCWs in training positions.

\section{References}

1. Pruss-Ustun, A., Rapiti, E., Hutin, Y. Estimation of the global burden of disease attributable to contaminated sharps injuries among healthcare workers. Am J Ind Med. 2005;48(6):482-90.

\section{UNAIDS. Report on the Global AIDS Epidemic. 2008(August).}

3. Musa OI. Injection safety practice among health workers in static immunization centres in a Nigerian urban community. Trop Doct. 2006;36:185-6.

4. Bryant J, Baxter L, Hird S. Non-occupational postexposure prophylaxis for HIV: a systematic review. Health Technol Assess. 2009;13(14):iii, ix-x, 1-60.

5. Cardo D, Culver D, Ciesielski C, e.a. A case-control study of HIV seroconversion in health care workers after percutaneous exposure. $N$ Engl J Med. 1997;337(21):1485-90.

6. Dufresne MD, Alfandari S, Fontier C, e.a. Tolerance, compliance and psychological consequences of post-exposure prophylaxis in healthcare workers. Int J Std AIDS. 1998;9(10):591-4

7. Otten RA, Smith DK, Adams DR, e.a. Efficacy of postexposure prophylaxis after intravaginal exposure of pig-tailed macaques to a human-derived retrovirus (human immunodeficiency virus type 2). $J$ Virol. 2000;74(20):9771-5.

8. Wade NA, Birkhead GS, Warren BL, e.a. Abbreviated regimens of zidovudine prophylaxis and perinatal transmission of the human immunodeficiency virus. N Engl J Med. 1998;339(20):1409-14.

9. Malawi Ministry ofHealth. Treatment ofAIDS, MalawiART guidelines 3rd ed. http://www.hivunitmohmw.org/Main/AntiretroviralTherapy.

10. van Oosterhout JJ, Nyirenda M, Beadsworth MB, e.a. Challenges in HIV post-exposure prophylaxis for occupational injuries in a large teaching hospital in Malawi. Trop Doct. 2007;37(1):4-6.

11. Ministry of Health and Population, Planning Department, Health Management Information Unit. Performance Data Analysis of Tertiary Care Facilities. Health Information Bulletin. 2003.

12. Lewis DK, Callaghan M, Phiri K, e.a. Prevalence and indicators of HIV and AIDS among adults admitted to medical and surgical wards in Blantyre, Malawi. Trans R Soc Trop Med Hyg. 97(1):91-6.

13. M'ikanatha NM, Imunya SG, Fisman DN, Julian KG. Sharp-device injuries and perceived risk of infection with bloodborne pathogens among healthcare workers in rural Kenya. Infect Control Hosp Epidemiol. 2007;28(6):761-3.

14. Nsubuga FM, Jaakkola MS. Needle stick injuries among nurses in sub-Saharan Africa. Trop Med Int Health. 2005;10(8):773-81.

15. Gumodoka B, Favot I, Berege ZA, Dolmans WMV. Occupational exposure to the risk of HIV infection among health careworkers in Mwanza region, United Republic of Tanzania. Bull World Health Organ. 1997;1997(75):133-40.

16. Taegtmeyer M, Suckling R, Nguku P, e.a. Working with risk: Occupational safety issues among healthcare workers in Kenya. AIDS Care. 2008;20(3):304-10.

17. Gounden YP, Moodley J. Exposure to human immunodeficiency virus among healthcare workers in South Africa. Int J Gynaecol Obstet. 2000;69(3):265-70.

18. Gupta A, Anand S, Sastry J, e.a. High risk for occupational exposure to HIV and utilization of post-exposure prophylaxis in a teaching hospital in Pune, India. BMC Infect Dis. 2008;8:142. 
19. Hiransuthikul N, Hiransuthikul P, Kanasuk Y. Human immunodeficiency virus postexposure prophylaxis for occupational exposure in a medical school hospital in Thailand. $J$ Hosp Infect. 2007;2007(67):344-9.

20. Tarantola A, Koumare A, Rachline A, e.a. Adescriptive, retrospective study of 567 accidental blood exposures in healthcare workers in three West African countries. J Hosp Infect. 2005;60(3):276- 82.

21. Davanzo E, Frasson C, Morandin M, Trevisan A. Occupational blood and body fluid exposure of university health care workers. Am J Infect Control. 2008;36(10):753-6.

22. Talaat M, Kandeel A, El-Shoubary W, e.a. Occupational exposure to needlestick injuries and hepatitis B vaccination coverage among health care workers in Egypt. Am J Infect Control. 2003;31(8):469-74

23. Nyirenda M, Beadsworth MBJ, Stephany P, e.a. Prevalence of infection with hepatitis $\mathrm{B}$ and $\mathrm{C}$ virus and coinfection with HIV in medical inpatients in Malawi. J Infect. 2008;57(1):72-77.

24. Aggarwal R, Ranjan P. Preventing and treating hepatitis B infection. Br Med J. 2004;329(7474):1080-1086.

25. Siika AM, Nyandiko WM, Mwangi A, e.a. The structure and outcomes of a HIV postexposure prophylaxis program in a high HIV prevalence setup in western Kenya. J. Acquir Immune Defic Syndr:2009;51(1):47-53.

Figure 4. PEP publicity campaign in $\mathrm{QECH}$

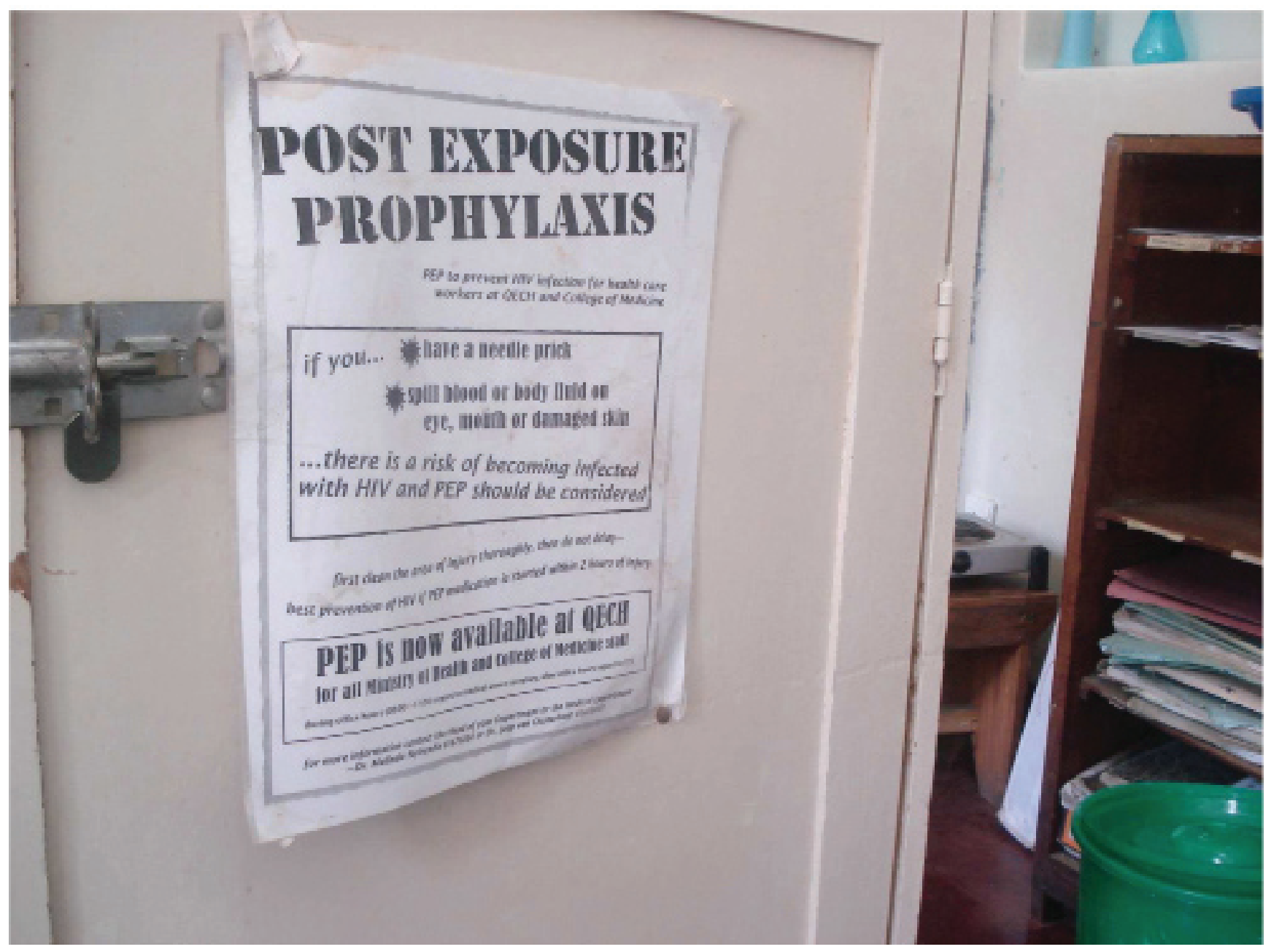

